Recepción: 20 / 04 / 2017

Aceptación: 20 / 05 / 2017

Publicación: 15 / 07 / 2017

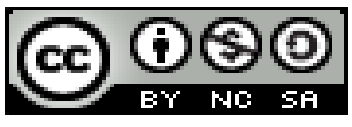

Ciencias de la Educación

Artículo Científico

\title{
La logopedia, procesos y funciones del lenguaje y comunicación humana
}

\section{Logopedia, processes and functions of human language and communication}

\section{Fonoaudiologia, processos e funções da linguagem \\ e da comunicação humana}

\author{
María D. Enireb-García ${ }^{\mathrm{I}}$ \\ maria.enirebg@ug.edu.ec \\ Fernando J. Jachero-Ochoa ${ }^{\text {II }}$ \\ fernando.jacheroo@ug.edu.ec \\ Viviana P. Patiño-Zambrano ${ }^{\text {III }}$ \\ viviana.patinoz@ug.edu.ec
}

Correspondencia: maria.enirebg@ug.edu.ec

\footnotetext{
Magister; Universidad de Guayaquil, Guayaquil, Ecuador. Docente, Universidad de Guayaquil, Guayaquil, Ecuador. Magister; Universidad de Guayaquil, Guayaquil, Ecuador.
} 


\section{Resumen}

Elaborar un plan de intervención logopédica que contribuya en la mejora de los procesos y funciones del lenguaje y la comunicación, de las personas con alteración del lenguaje, causado por un accidente cerebro vascular (ACV), también llamado ictus; que asisten al Centro de Atención a la Comunidad de la Facultad de Ciencias Médicas, de la UG. El ACV es una discapacidad multicausal de gran alcance biopsicosocial, como lo revelan las estadísticas expuestas en los diferentes países. El estudio de caso que se presenta se realizó en un paciente de 51 años, con afasia de Broca, con evaluación pre y postratamiento. Por su naturaleza, la investigación es de transferencia metodológica y de un diseño técnico correspondiente al campo de la salud. Se empleó el Método Clínico y el Método de Asociación de Mildred McGinnis. Durante 14 semanas se aplicó un programa basado en las dificultades articulatorias identificadas durante la evaluación inicial, dividido en tres momentos, con un objetivo específico cada una. Resultados: Se observó aumento en la habilidad para articular fonemas, disminución en las parafasias literales y un incremento en el lenguaje expresivo verbal del sujeto. Conclusión: La valoración neuropsicológica y el diseño de la intervención y tratamiento logopédico ayudaron a corregir las dificultades de punto y modo de articulación, observadas en este sujeto; así como el incremento de las dimensiones del lenguaje más complejas.

Palabras claves: Logopedia; estudio de caso; método de asociación de mildred mcginnis; afasia de broca. 


\section{Abstract}

To elaborate a plan of intervention logopédica that contributes in the improvement of the processes and functions of the language and the communication, of the people with alteration of the language, caused by a stroke (stroke), also called stroke; Who attend the Community Care Center of the Faculty of Medical Sciences, UG. LCA is a multi-causal, biopsychosocial disability, as shown by the statistics presented in different countries. The case study presented was performed in a 51 -yearold patient with Broca's aphasia, with pre- and post-treatment evaluation. By its nature, the research is of methodological transfer and of a technical design corresponding to the field of health. The Clinical Method and the Method of Association of Mildred McGinnis were used. During 14 weeks, a program based on the articulatory difficulties identified during the initial evaluation was applied, divided into three moments, with a specific objective each. Results: There was an increase in the ability to articulate phonemes, a decrease in literal paraphasias and an increase in the verbal expressive language of the subject. Conclusion: The neuropsychological assessment and the design of the intervention and speech therapy helped to correct the difficulties of point and mode of articulation, observed in this subject; As well as increasing the more complex language dimensions.

Key words: Speech therapy; case study; method of association of mildred mcginnis; drill aphasia. 


\section{Resumo}

Desenvolver um plano de intervenção fonoaudiológica que contribui para a melhoria dos processos e funções da linguagem e comunicação, pessoas com distúrbios de linguagem, causado por um acidente vascular cerebral (AVC), também chamado de acidente vascular cerebral; atendendo Centro de Atendimento à Comunidade da Faculdade de Ciências Médicas, UG. LCA é uma grande deficiência biopsicossocial multicausal alcançar, como revelado pelas estatísticas apresentadas em diferentes países. O estudo de caso apresentado foi realizado em um paciente de 51 anos com afasia de Broca, com avaliação pré e pós tratamento. Por sua natureza, a investigação é uma transferência metodológica e desenho técnico para a área da saúde. método clínico e Associação de Mildred McGinnis foi usada. Durante 14 semanas com base nas dificuldades articulatórias identificados durante o programa de avaliação inicial, divididos em três fases, com um alvo específico cada aplicado. Resultados: Foi observada aumento da capacidade de articular fonemas, diminuiu parafasias literais e aumento da linguagem expressiva verbal do assunto. Conclusão: A avaliação neuropsicológica e intervenção de design e terapia da fala ajudou a corrigir as dificuldades e os tipos de ponto de articulação, observado neste assunto; bem como o aumento do tamanho da linguagem mais complexa.

Palavras-chave: Terapia da fala; método de estudo de caso e de associação Mildred McGinnis da afasia de Broca. 


\section{Introducción.}

La Universidad de Guayaquil (UG) se encuentra preocupada en responder a los cambios sociales, económicos, culturales y políticos que acontecen en el país, en concordancia con el interés del Estado, la comunidad latinoamericana y mundial. Esta situación insoslayable requiere una profunda renovación que dé paso a la transformación del proceso enseñanza-aprendizaje, al incremento de la investigación científica, a la implementación de su infraestructura y presentación continua de proyectos vinculados a la comunidad, realizados con tino y veracidad.

Junto a esta renovación debe estar presente el reencuentro con nuestras raíces culturales; que permita incorporar y recuperar los códigos de nuestros ancestros. El Sumak Kawsay y el Sumak Qamaña (Vivir en Plenitud, con el sustento del trabajo y el conocimiento) son testimonio de valores humanos imprescindibles en la práctica de la vida diaria, porque a través de ellos se actúa con autenticidad, sentido patrio y transparencia. Estos valores morales son referentes para las generaciones ávidas de justicia social, equidad y cumplimiento de los derechos humanos.

El Proyecto ofrece a la comunidad una propuesta que favorece la actividad de los procesos y funciones del lenguaje y comunicación, desde las bases del sistema de Atención Primaria de Salud (APS); a fin de paliar, recuperar y resolver los problemas de las deficiencias y discapacidades lingüísticas, de la deglución y tubáricas, en sujetos de todas las edades, abordados de manera holística en el marco del enfoque biopsicosocial, axiológico, ambientalista y solidario, con la intención de mejorar el estado de salud de la población, en el ámbito del cumplimiento del Plan Nacional del Buen Vivir. 


\section{Materiales y métodos.}

Esta investigación se lleva a cabo utilizando el método científico como base fundamental de los siguientes métodos:

\section{Métodos teóricos.}

Método clínico

Estudio de casos

\section{Métodos empíricos}

\section{Análisis documental.}

Método de Asociación de Mildred McGinnis: para promover las funciones del lenguaje y la comunicación, que en este caso en paciente con afasia de Broca.

Métodos estadísticos: se utilizó el método de análisis porcentual para comprobar la efectividad de la propuesta aplicada.

Aporte a la práctica: sistema de acciones de intervención logopédica en paciente con afasia de broca. (gooldstein; afasia motora; head: afasia verbal; luria: afasia motora eferente).

\section{Caracterización del Paciente}

Se llevó a cabo en un estudio de caso en un paciente de 51 años, con afasia de Broca, inteligencia normal, quien se presenta solo a la consulta, luego de un segundo episodio vascular cerebral, el primero acaecido hace un año y el segundo hace cinco meses. Se realiza por primera vez tratamiento logopédico, con la respectiva evaluación pre y postratamiento. Su lenguaje se 
caracteriza por torpeza articular motora, limitación del vocabulario, restricción gramatical a las formas más simples y sobreaprendidas, con omisión de palabras obligatorias. La comprensión auditiva se encuentra conservada.

La orientación temporal y espacial, a pesar de que no es capaz de expresar oralmente la oración completa, responde cuales son los días de la semana y los meses del año, así como es capaz de responder a preguntas del tipo: ¿qué día es hoy?, ¿Cuándo viene a la rehabilitación?, ¿Cuándo es su cumpleaños? En conclusión, el paciente puede referirse a prácticamente a los problemas de la vida diaria con ayuda. Sin embargo, la reducción del habla y/o la comprensión se hacen sumamente difícil en especial la comprensión sobre cierto tipo de temas. El lenguaje escrito sigue el patrón del lenguaje oral, ya que la lectura se encuentra levemente afectada; mientras que la escritura se presenta moderadamente alterada, debido a una parecía del lado derecho. La comprensión de palabas aisladas no se encuentra afectada, pero sí, la dificultad con sintaxis más compleja. (Ver historia Clínica, apéndice B).

\section{Intervención y tratamiento logopédico}

El tratamiento se inicia, luego de aplicar la anamnesis y el Test de Boston para el Diagnóstico de la Afasia. Láminas de estímulo. Formato abreviado (2005), 3era. Ed. Una vez obtenido el perfil neuropsicológico del paciente, se establece un diseño terapéutico que contempla la aplicación del Método de Asociación de Mildred McGinnis. Se utiliza el lenguaje repetido, como herramienta de valoración de la estimulación de la repetición, técnica de enseñanza aprendizaje; y el lenguaje espontáneo, como elemento que enriquecerá sustancialmente la información obtenida mediante lenguaje inducido, orientado a valorar la pragmática. La intervención se encamina hacia procesos generales, trabajando todos los segmentos afectados, centrándonos primero en la elicitación 
fonoarticulativa (por ejemplo, dice "geo" por reloj; la mañana esta soleada,- dice- añana soada), para continuar con la inestabilidad de la producción y en la eliminación de homónimos, yendo desde el menor conocimiento al de mayor conocimiento para ir incrementando paulatinamente el lenguaje expresivo del paciente, que en muchas expresiones el lenguaje se muestra con estereotipias.

De la fase de diagnóstico del estado neuropsicológico funcional, se elabora el pretest (línea de base), para luego realizar la segunda fase (tratamiento), en que se procedió con la Valoración fonética: punto y modo de articulación, para identificar los fonemas que afectan función del habla. Se utiliza la clasificación de Santos Borregón (2010). En la tercera fase se procede al postest, para evaluar la eficacia del tratamiento.

Cuadro $\mathbf{N}^{\circ}$ 2. Valoración del Vocabulario (línea base).

\begin{tabular}{|llllll|}
\hline Pares de palabras & ejecución & palabras & ejecución & Sílabas & ejecución \\
Gata - lata & gata-gata & árbol & abon & ta-ma & pa-da \\
Moño- dona & doña-cona & plátano & patano & sa-la & ca-sa \\
Rosa- roma & bosa-bena & blusa & bunsa & ma-ma & da-da \\
Trompo-tozo & tonpo-tonpo & lindo & cindo & la-la & no-na \\
\hline
\end{tabular}

Cuadro $\mathbf{N}^{\circ}$ 3. Fonemas identificados

\begin{tabular}{|l|l|l|l|}
\hline Fonemas & Modo de Articulación & Punto de Articulación & Acción de las cuerdas vocales \\
\hline $\begin{array}{l}\mathbf{R} \\
\mathbf{R}\end{array}$ & $\begin{array}{l}\text { vibrante simple } \\
\text { vibrante múltiple }\end{array}$ & $\begin{array}{l}\text { apicoalveolar } \\
\text { apicoalveolar }\end{array}$ & $\begin{array}{l}\text { sonoro bucal } \\
\text { sonoro bucal }\end{array}$ \\
\hline $\mathbf{L}$ & lateral & apicoalveolar & sonoro bucal \\
\hline $\mathbf{M}$ & nasal & bilabial & sonoro bucal \\
\hline
\end{tabular}

Cuadro $\mathrm{N}^{\circ} 4$ Pretest: Porcentaje de aciertos en praxias bucofonoarticulatorias. 
La logopedia, procesos y funciones del lenguaje y comunicación humana

\begin{tabular}{|l|c|c|c|}
\hline \multicolumn{1}{|c|}{$\begin{array}{c}\text { Categorías } \\
\mathbf{R}-\mathbf{L}-\mathbf{M}\end{array}$} & $\begin{array}{c}\text { Aciertos } \\
\mathbf{1 0 0 \%} \mathbf{- 8 0} \%\end{array}$ & $\begin{array}{c}\text { Aciertos } \\
\mathbf{7 9} \boldsymbol{\%}-\mathbf{5 0} \%\end{array}$ & $\begin{array}{c}\text { Aciertos } \\
-\mathbf{4 9} \%\end{array}$ \\
\hline Praxias linguales & & & $40 \%$ \\
\hline Praxias labiales & & $60 \%$ & \\
\hline $\begin{array}{l}\text { Praxias mandibulares } \\
\text { y velo del paladar }\end{array}$ & & $60 \%$ & \\
\hline
\end{tabular}

\section{Resultados.}

Resultados de la segunda valoración: Luego del aprendizaje de la primera unidad que comprende la praxis fonoarticulatoria y 50 sustantivos (lectura, escritura y dictado de palabras) así como las respuestas a preguntas que dan inicio a la segundad unidad del Método de Asociación, (aprendizaje de verbos, pronombres, elaboración de preguntas y respuestas, etc). Se puede decir, que el lenguaje espontáneo del paciente mejoró en comparación con lo observado en la primera valoración (tablas 1 y 2), así como en su precisión de pronunciación, superando en un $90 \%$ el rotacismo, lambdacismo y mitacismo,- tal como se demuestra en la tabla que se describe a continuación, aunque que las parafasias literales no se eliminaron del todo.

Cuadro $\mathbf{N}^{\circ}$ 5. Postest: Porcentaje de aciertos en praxias bucofonoarticulatorias

\begin{tabular}{|l|c|c|c|}
\hline \multicolumn{1}{|c|}{$\begin{array}{c}\text { Categorías } \\
\text { R }-\mathbf{L}-\mathbf{M}\end{array}$} & $\begin{array}{c}\text { Aciertos } \\
\mathbf{1 0 0} \mathbf{\%}-\mathbf{8 0} \%\end{array}$ & $\begin{array}{c}\text { Aciertos } \\
\mathbf{7 9} \boldsymbol{\%} \mathbf{5 0} \%\end{array}$ & $\begin{array}{c}\text { Aciertos } \\
-\mathbf{4 9} \%\end{array}$ \\
\hline Praxias linguales & $80 \%$ & & \\
\hline Praxias labiales & $85 \%$ & & \\
\hline Praxias mandibulares y velo del paladar & $90 \%$ & & \\
\hline
\end{tabular}


Las estrategias del diseño metodológico, confirma la premisa: el Método de Asociación de Mildred McGinnis mejora las funciones del lenguaje y la comunicación de las personas con afasia de Broca, de manera eficiente. En el caso que nos ocupa mejoró la actividad verbo-bocal. Se superó en las praxias linguales $100 \%$; en las praxias labiales un 42\%; y 50\% en las mandibulares y velo del paladar, lo cual garantiza la mejora del paciente en términos de lograr una recuperación de la pragmática necesaria para integrarse en su vida social.

\section{Conclusiones.}

Los problemas originados por una deficiente cultura organizacional; escasos programas de educación continua; inexistentes estudios de cuarto nivel orientados a formación profesional del logopeda; la infraestructura precaria en que se desenvuelve el docente y el estudiante, impiden el avance del proceso enseñanza aprendizaje y afecta en extensión, profundidad y rigor el proceso académico. Estos factores no son más que un reflejo del deterioro global por el que atraviesa la UG, que repercuten en la carrera Terapia del Lenguaje.

Por primera ocasión se presenta un sistema de acciones concretas para el tratamiento de personas con afasia de Broca, aplicando el Método de Asociación de Mildred McGinnis. De esta manera se exalta la importancia de investigar en el medio natural donde se desenvuelve la carrera; más aún, se evidencia la interacción entre estudiantes supervisados por docentes especializados en la atención de los casos con periodicidad señalados por el profesional responsable de la intervención logopédica. 


\section{Recomendaciones.}

- Motivar en las autoridades de la UG, la necesidad de programas de educación continua y estudios de cuarto nivel relacionados a la ciencia logopedia, para elevar los estándares de calidad del docente universitario y del ejercicio profesional en general

- Asignar los recursos necesarios para implementar la infraestructura en todos los campos del conocimiento de la carrera.

- Sugerir el cambiar de la nomenclatura de la carrera TL, por la de Logopedia. La logopedia es una ciencia, la terapia del lenguaje es la praxis de esa ciencia, por esta condición y carácter integrador de otras disciplinas vinculadas a los procesos y funciones del lenguaje y comunicación, se encuentra con mayor capacidad de resolver los problemas del encargo social. Más aún, no se encuentra registrada la nomenclatura Terapia del Lenguaje en los Códigos CINE de la UNESCO.

\section{Bibliografía}

Álvarez, c. (2010). La relación entre lenguaje y pensamiento de Vigostki en el desarrollo de la psicolinguística moderna. Rla. Revista de linguística teórica y aplicada, 48(2), 13-32. Https://doi.org/10.4067/s0718-48832010000200002

Ardila, A. (2005). Las afasias (primera). México, Guadalajara: pandora. Recuperado a partir de: http://www.jmunozy.org/files/9/logopedia/afasia tcv/documentos/bv afasias.pdf

Ardila, R. (2011). Inteligencia. ¿Qué sabemos y qué nos falta por investigar? Revista de la academia colombiana de ciencias exactas, físicas y naturales, 35(134), 97-103.

Ardila, A. (2013). Trastornos del lenguaje en pacientes con lesiones cerebrales. Florida International University Miami, Florida, EE.UU. Recuperado a partir de https://aalfredoardila.files.wordpress.com/2013/07/2013-ardila$\underline{\text { trastornos del lenguaje en pacientes con lesiones-cerebrales.pdf }}$

Arteaga, J. (2010). El método clínico y el método científico.

Medisur 2010 e-ISSN: 1727-897x. Revista electrónica de las Ciencias Médicas en Cienfuegod, Cuba.

Barriga L., F. (1982). La educación especial en el ecuador. Casa de la cultura ecuatoriana "Benjamín Carrión" núcleo del guayas. 
Beber, E.R., (2012). Método epidemiológico. Universidad de San Carlos de Guatemala. Https://saludpublica1.files.wordpress.com/2012/08/mc3a9todo-epidemiolc3b3gico.pdf

Becerra, C.,(2008). Lenguaje y educación en niños sordos: encuentros y desencuentros. Rexe. Revista de estudios y experiencias en educación. Issn: 0717-6945. Rexe@ucsc.cl. Redalyc.org/articulo.oa? $=243117031007$

Bernal, C. A., (2010). Metodología de la investigación. Administración, economía, humanidades y ciencias sociales (tercera). Colombia: pearson education.

Recuperado a partir de http://eva.sepyc.gob.mx:8383/greenstone3/sites/localsite/collect/ciencia1/index/assoc/hashe5b1.dir/11050004 .pdf

Borregón Sanz, S. (2010). Los trastornos de la articulación. Exploración, prevención, diagnóstico y tratamiento. Madrid, España: ciencias de la educación preescolar y especial.

Calzadilla, R. (2004). La pedagogía como ciencia humanista: conocimiento de síntesis, complejidad y pluridisciplinariedad. Revista de pedagogía, 25(72), 123-148.

Cháves S., A. (2001). Implicaciones educativas de la teoría sociocultural de Vigotsky. Revista educación, 25(2), 59-65.

Cosacov, E., (2005). Introducción a la psicología (5ta.). Argentina: brujas.

Cruz,M., Fumero, C., González A., (2016). Abordaje de la comunicación en un caso de afasia Global. International journal of developmental and educational psychology Infad revista de psicología, nº 2, 2016. Issn: 0214-9877. Pp: 267-276 https://doi.org/10.17060/ijodaep.2016.n2.v1.555

Enireb, m., (1999). Terapia del lenguaje (1era.). Guayaquil. Ecuador: universidad de Guayaquil.

Escamilla G., A., \& Javierre G., M. (2003). Historia i. De la prehistoria a los albores de la sociedad industrial. En enciclopedia del conocimiento (vol. 1). Espasa calpe.

Fernández p., g. (s/f). Enfoque cubano de atención a los trastornos de la comunicación y el lenguaje. Actualidad y perspectivas. Recuperado el 29 de agosto de 2016, a partir de http://www.sld.cu/galerias/pdf/sitios/rehabilitacion-

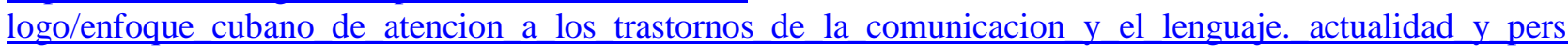
pectivas.pdf

Figueredo, Ernesto. (1984). Logopedia 1. La Habana: pueblo y educación.

Fuentealba Weber, L. (1986). Revista de sociología. Departamento de sociología - facultad de ciencias sociales. Universidad de chile. Https://doi.org/10.5354/0719-529x.1986.27541

Galindo Aldana, Pelayo González, H., Solovieva, Y., \& Quintanar Rojas, L. (2014). Rehabilitación neuropsicológica en un caso de afasia motora aferente. Pensamiento psicológico, 12(2), 97-112.

García r., r., \& González r., v. (1988). Las funciones psíquicas superiores, la corteza cerebral y la cultura. Reflexiones a partir del pensamiento de a. R. Luria en-claves del pensamiento, vol. Viii, núm. 15, enero- 
junio, 2014, pp. 39-62 inst, viii. Recuperado a partir de http://www.sld.cu/galerias/pdf/sitios/rehabilitacion$\underline{\text { logo/historia de la logopedia y foniatria.pdf }}$

Gómara, A., Amezana, M., El estudio de casos. Un instrumento de aprendizaje en la Relación de cuidado. Versión on-line ISSN 1699-5988versión impresa ISSN 1132- 1296. Index enferm vol.23 no.4 granada oct./dic. 2014

González LP; González OB. (2012). Afasia: de la teoría a la práctica (primera). México. 